\title{
High-Resolution DCE-MRI of the Pituitary Gland Using Radial k-Space Acquisition with Compressed Sensing Reconstruction
}

\author{
M.C. Rossi Espagnet, (DL. Bangiyev, M. Haber, K.T. Block, J. Babb, V. Ruggiero, F. Boada, O. Gonen, and G.M. Fatterpekar
}

\begin{abstract}
BACKGROUND AND PURPOSE: The pituitary gland is located outside of the blood-brain barrier. Dynamic T7 weighted contrast enhanced sequence is considered to be the gold standard to evaluate this region. However, it does not allow assessment of intrinsic permeability properties of the gland. Our aim was to demonstrate the utility of radial volumetric interpolated brain examination with the golden-angle radial sparse parallel technique to evaluate permeability characteristics of the individual components (anterior and posterior gland and the median eminence) of the pituitary gland and areas of differential enhancement and to optimize the study acquisition time.
\end{abstract}

MATERIALS AND METHODS: A retrospective study was performed in 52 patients (group 1, 25 patients with normal pituitary glands; and group 2, 27 patients with a known diagnosis of microadenoma). Radial volumetric interpolated brain examination sequences with goldenangle radial sparse parallel technique were evaluated with an ROI-based method to obtain signal-time curves and permeability measures of individual normal structures within the pituitary gland and areas of differential enhancement. Statistical analyses were performed to assess differences in the permeability parameters of these individual regions and optimize the study acquisition time.

RESULTS: Signal-time curves from the posterior pituitary gland and median eminence demonstrated a faster wash-in and time of maximum enhancement with a lower peak of enhancement compared with the anterior pituitary gland $(P<.005)$. Time-optimization analysis demonstrated that 120 seconds is ideal for dynamic pituitary gland evaluation. In the absence of a clinical history, differences in the signal-time curves allow easy distinction between a simple cyst and a microadenoma.

CONCLUSIONS: This retrospective study confirms the ability of the golden-angle radial sparse parallel technique to evaluate the permeability characteristics of the pituitary gland and establishes 120 seconds as the ideal acquisition time for dynamic pituitary gland imaging.

ABBREVIATIONS: GRASP = golden-angle radial sparse parallel; STC = signal-time curve; VIBE = volumetric interpolated brain examination

$\mathrm{T}$ he pituitary gland is a highly perfused gland located within the sella turcica and outside the blood-brain barrier. Pathologies intrinsic to this region, listed from most common to least common, include benign micro- and macroadenomas, invasive adenomas, and carcinomas. Of these, benign pituitary adenomas represent $10 \%-25 \%$ of all intracranial neoplasms, with an estimated prevalence rate of $17 \%$ within the general population. ${ }^{1}$

Received November 20, 2014; accepted after revision January 6, 2015

From the Department of Radiology, New York University Langone Medical Center, New York, New York.

Paper previously presented in part at: Annual Meeting of the American Society of Neuroradiology and the Foundation of the ASNR Symposium, May 17-22, 2014;

Montreal, Quebec, Canada.

Please address correspondence to M.C. Rossi Espagnet, MD, Department of Radiology, New York University Langone Medical Center, 550 First Ave, New York, NY 10016; e-mail: camilla.rossiespagnet@gmail.com

- Indicates open access to non-subscribers at www.ajnr.org

http://dx.doi.org/10.3174/ajnr.A4324
MR imaging is the criterion standard for the evaluation of the pituitary gland. Imaging is performed in a dynamic manner by using section-selective T1-weighted TSE sequences before and at multiple time points after the injection of a gadolinium-based contrast agent. ${ }^{2,3}$ Such dynamic scanning allows assessment of underlying pathology, especially microadenomas, by evaluating any focal area of differential enhancement within the pituitary gland. However, such a dynamic scanning technique has certain limitations. Enhancement within the posterior pituitary gland cannot be appreciated due to its inherently bright T1 signal. Evaluation of very small-sized (1-3 mm) microadenomas can be challenging, depending on the underlying section thickness. Distinction between a simple cyst and a microadenoma can sometimes be difficult, especially without a good history. Furthermore, there is not a standard dynamic image-acquisition timeframe. Literature suggests that the acquisition time for the dynamic sequence varies among different institutions, ranging from 150 to 240 seconds. ${ }^{4-6}$ 
Standardization of this image acquisition time is especially important in today's economic imaging scenario, where we strive for the best possible information in the most appropriate time.

Golden-angle radial sparse parallel MR imaging (GRASP) is a new volumetric dynamic imaging technique based on a $3 \mathrm{D}$ gradient-echo sequence with radial "stack-of-stars" $k$-space sampling ${ }^{7}$ and golden-angle ordering. ${ }^{8}$ As opposed to the conventional dynamic MR imaging techniques that perform multiple separate examinations, the GRASP technique acquires all dynamic information in a single continuous scan during which the contrast agent is injected. Image reconstruction is then achieved by binning the data into sequential timeframes and reconstructing the frames with an iterative method that combines parallel imaging and compressed sensing. ${ }^{9}$ By using a total-variation constraint along time, the GRASP technique can reconstruct images from highly undersampled data, offering simultaneously high submillimeter spatial and excellent temporal resolution. In addition, a unique feature of GRASP imaging is that the desired temporal resolution can be selected retrospectively and can be designated as high as approximately 2.5 seconds per frame. Thus, compared with conventional 2D TSE examinations, GRASP provides greater through-plane resolution, improved sensitivity to motion and flow, and improved fat suppression. ${ }^{10}$

The purpose of our study was, therefore, to evaluate the role of GRASP for assessing the pituitary gland. More specifically, the study proposed to do the following: 1) establish normal enhancement patterns and signal-time curves (STCs) for the anterior and posterior pituitary gland and median eminence, 2) optimize the acquisition time for dynamic pituitary gland imaging, and 3) evaluate differences in the STCs to distinguish a simple cyst from a microadenoma.

\section{MATERIALS AND METHODS \\ Patients}

This study was approved by the institutional review board and is Health Insurance Portability and Accountability Act-compliant. We retrospectively evaluated MR imaging data of 52 patients who underwent dynamic imaging of the pituitary gland by using the GRASP technique between July 2013 and November 2013. For our study, these patients were divided into 2 groups: group 1 patients $(n=25$; male/female ratio, 9:16; age range, $10-74$ years; mean age, 39 years) included healthy volunteers $(n=8)$ or patients undergoing brain studies for the evaluation of headache $(n=13)$ and patients who had undergone pituitary gland studies for incidentally noted suspicious sellar lesions on prior conventional brain studies $(n=4)$. Group 2 consisted of 27 patients (male/female ratio, 12:15; age range, 26-63 years; mean age, 37 years) with a known microadenoma. Specifically, patients were included in group 2 if they had the following: 1) a history of endocrinologic disturbances favoring a central cause, and 2) previous MR imaging studies demonstrating a focus of "reduced" differential enhancement within the pituitary gland compatible with a microadenoma. Patients with susceptibility artifacts at the skull base resulting from dental hardware $(n=2)$ and aneurysm clips $(n=1)$ were excluded from the study. Patients with hem- orrhagic $(n=1)$ or cystic lesions $(n=2)$ within the pituitary gland were also excluded.

\section{MR Imaging}

All patients underwent MR imaging with a 3T system (Magnetom Skyra; Siemens, Erlangen, Germany). A 20-channel head/neck coil was used. Imaging protocol included a coronal radial volumetric interpolated brain examination (VIBE) with a GRASP acquisition (TR/TE, 6.4/2.4 ms; in-plane resolution, $0.7 \mathrm{~mm}$; section thickness, $0.8 \mathrm{~mm}$; 32 sections; FOV, $180 \mathrm{~mm}$; flip angle, 9.5 bandwidth, $391 \mathrm{~Hz} /$ pixel; pixel base resolution, 256; 800 spokes; acquisition time, 180 seconds), precontrast sagittal T1 (TR/TE, 440/2.66 ms; section thickness, 3 mm; 25 sections; FOV, 160 mm; flip angle, $90^{\circ}$; bandwidth, $380 \mathrm{~Hz} /$ pixel; pixel base resolution, 320), coronal T2 (TR/TE, 4000/97 ms; section thickness, 2 mm; 15 sections; FOV, $140 \mathrm{~mm}$; flip angle, 150 ; bandwidth, $260 \mathrm{~Hz} /$ pixel; pixel base resolution, 320), and axial FLAIR (TR/TE, 9000/90 ms; TI, 2500; section thickness, 5 mm; 15 sections; FOV, $220 \mathrm{~mm}$; flip angle, $150^{\circ}$; bandwidth, $290 \mathrm{~Hz} /$ pixel; pixel base resolution, 320). Contrast material, 0.01-mmol gadopentetate dimeglumine (Magnevist; Bayer HealthCare Pharmaceuticals, Wayne, New Jersey) per kilogram of body weight, was administered at $3 \mathrm{~mL} / \mathrm{s}$ on initiation of the GRASP sequence.

\section{Image Data Analysis and Processing}

Images were evaluated by 1 neuroradiologist (G.M.F., with 15 years of experience), who was blinded to the study population. The acquired data from the GRASP scans were exported and reconstructed off-line by using a $\mathrm{C}++$ implementation of the GRASP algorithm, creating 9 dynamic image frames with a temporal resolution of 20.00 seconds each. Reconstructed images were sent to the PACS and analyzed by using the software Olea Sphere, Version 2.2 (Olea Medical, La Ciotat, France) to obtain signal-time curves and generate permeability measurements, including wash-in, washout, peak, time to maximum enhancement, and area under the curve (Fig 1).

Evaluation of the normal-appearing pituitary gland was performed in group 1 by placing ROIs on the GRASP images of the anterior and posterior pituitary gland and the median eminence. Sagittal reconstructions confirmed the placement of ROIs within their respective locations (Fig $2 A-C$ ). The ROIs were used to generate STCs for each of these individual regions (Fig $2 D$ ). In addition, ROIs were placed within incidentally noted cysts $(n=3)$ in group 1 to compare the generated STCs with microadenomas in group 2.

To optimize image acquisition times, we generated STCs and permeability measurements for group 2, placing the ROIs in the normal-appearing pituitary gland and in the microadenoma (Fig 3).

\section{Statistical Analysis}

Statistical analysis was performed with SAS 9.3 software (SAS Institute, Cary, North Carolina). The analysis was divided into 2 parts. The first part was using the paired-sample Wilcoxon signed rank test to evaluated differences in normal STCs and permeability parameters of the anterior and posterior pituitary gland and 




FIG 1. Analysis of radial VIBE images provide maps of different permeability parameters: peak, area under the curve, time of maximum enhancement, wash-in, and washout.

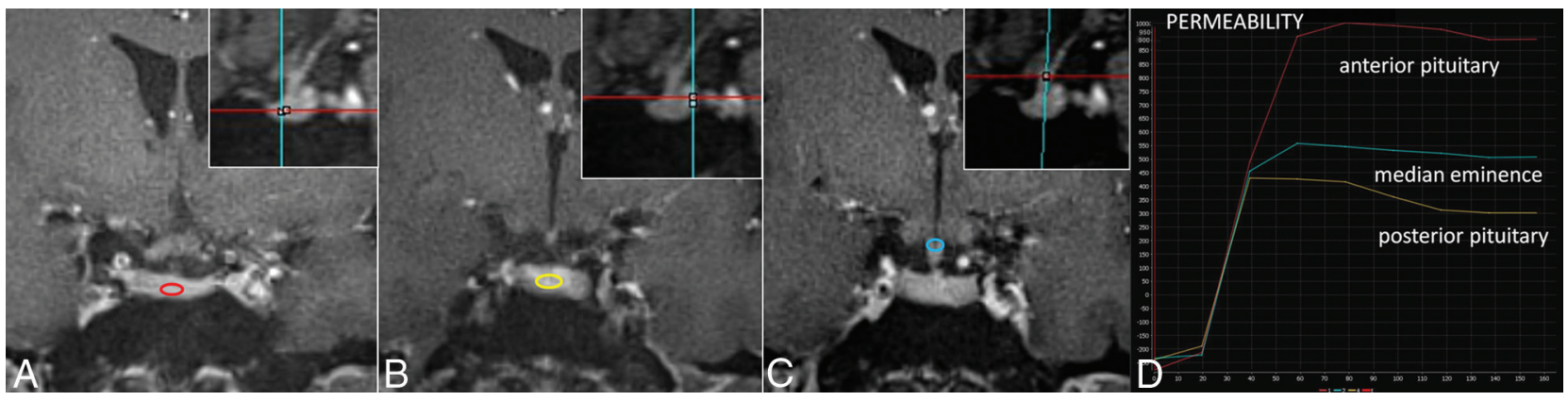

FIG 2. GRASP images in a healthy volunteer show the ROIs in the anterior pituitary gland ( $A$ ), posterior pituitary gland ( $B$ ), and median eminence (C). Sagittal reference planes are shown top right. Corresponding signal-time curves from the ROIs $(D)$ demonstrate a different pattern of enhancement in the anterior (red) and posterior pituitary gland (yellow) and the median eminence (light blue).

the median eminence from group 1 subjects. The mean signifi-

Table 1: Mean, SD, median, maximum, and lower and upper limits of a $95 \%$ confidence interval of the mean for the percentage change in the peak enhancement estimate from time T0 $=60$ seconds to time T8 $=140$ seconds in the normal-appearing anterior pituitary gland of group 2 patients

\begin{tabular}{clccccc}
\hline Time & Mean & SD & Median & Maximum & Lower & Upper \\
\hline 1 & 9.82 & 9.97 & 5.25 & 30.99 & 5.16 & 14.49 \\
2 & 6.11 & 6.65 & 4.52 & 21.69 & 3.00 & 9.23 \\
3 & 2.00 & 2.52 & 1.05 & 8.53 & 0.82 & 3.18 \\
4 & 2.17 & 2.55 & 1.18 & 8.98 & 0.97 & 3.36 \\
5 & 1.41 & 0.87 & 1.06 & 2.76 & 1.01 & 1.82 \\
6 & 1.38 & 0.87 & 1.04 & 3.82 & 0.98 & 1.79 \\
7 & 0.94 & 0.87 & 0.71 & 3.28 & 0.53 & 1.35 \\
8 & 0.57 & 0.33 & 0.62 & 1.30 & 0.42 & 0.73 \\
\hline
\end{tabular}

cance level was set up at $P<.005$. The second part of the analysis used the Student $t$ test and focused on optimizing the acquisition time for dynamic pituitary gland imaging. This was performed by comparing peak enhancement values between the anterior pituitary gland and the microadenoma in group 2 patients at 10 second time intervals beginning at 60 seconds (when the maximum enhancement was almost reached) to 140 seconds. This allowed us to estimate a total of 8 mean, minimum, and maximum percentage-change values beginning at T1 (from 60 to 70 seconds) until T8 (from 130 to 140 seconds) (Table 1). A Student $t$ test was used to demonstrate any significant difference in peak enhancement values between the normal-appearing anterior pituitary gland and the microadenoma in this time $(60-140 \mathrm{sec}-$ onds). The mean significance level was set at $P<.001$. 
Table 2: Values of perfusion parameters obtained from ROI analysis in different regions of the normal-appearing pituitary gland ${ }^{a}$

\begin{tabular}{|c|c|c|c|c|c|c|}
\hline \multirow[b]{2}{*}{ Parameter } & \multicolumn{2}{|c|}{ Anterior } & \multicolumn{2}{|c|}{ Posterior } & \multicolumn{2}{|c|}{ Median Eminence } \\
\hline & Mean & SD & Mean & SD & Mean & SD \\
\hline AUC & 69980.65 & 20169.32 & 37500.10 & 17701.73 & 40246.39 & 16626.70 \\
\hline Peak & 754.94 & 242.04 & 439.61 & 205.67 & 446.62 & 192.66 \\
\hline TME (sec) & 86.55 & 19.23 & 58.96 & 16.23 & 62.60 & 18.70 \\
\hline Wash-in & 10.92 & 3.89 & 9.14 & 4.20 & 8.54 & 3.71 \\
\hline Washout & 1.52 & 1.34 & 1.90 & 1.48 & 1.56 & 1.27 \\
\hline
\end{tabular}

Note:-TME indicates time of maximum enhancement

a All parameters other than TME are dimensionless.

\begin{tabular}{|c|c|c|c|}
\hline Time (sec) & Mean PE & Min PE & Max PE \\
\hline 60 & 327 & 90 & 500 \\
\hline 70 & 365 & 120 & 600 \\
\hline 80 & 400 & 170 & 630 \\
\hline 90 & 420 & 200 & 660 \\
\hline 100 & 417 & 180 & 650 \\
\hline 110 & 342 & 165 & 625 \\
\hline 120 & 345 & 172 & 620 \\
\hline 130 & 346 & 171 & 613 \\
\hline 140 & 344 & 170 & 613 \\
\hline
\end{tabular}

Note:-PE indicates peak enhancement; Min, minimum; Max, maximum.

\section{RESULTS}

\section{Region Analysis (Group 1)}

An evaluation of the STCs derived from the anterior and posterior pituitary gland and the median eminence demonstrated differential patterns of enhancement for each of these individual regions. STCs from the posterior pituitary gland and the median eminence demonstrated a faster wash-in (9.14 and 8.54, respectively) compared with the anterior pituitary gland (10.92), a faster time of maximum enhancement (58.96 and 62.60 seconds, respectively) compared with that of the anterior pituitary gland (86.55 seconds), and a lower peak of enhancement (439.61 and 446.62, respectively) compared with the anterior pituitary gland (754.94). The anterior pituitary gland and median eminence showed a faster washout compared with the posterior pituitary gland (1.52 and 1.56 versus 1.90 , respectively). The results of the statistical analysis of the preceding data are summarized in Table 2. These findings are consistent across patients in group 1, with a mean significance level of $P<.005$.

\section{Time Analysis (Group 2)}

The mean peak enhancement for the anterior pituitary gland was reached at $80 \pm 10$ seconds. There was no significant change in the enhancement values for the anterior pituitary gland after 90 seconds (Table 1). The mean peak enhancement value for the microadenoma was reached at $90 \pm 10$ seconds. There was a relative plateau to mild washout noted in the enhancement values measured subsequent to the peak enhancement time (Table 3). A statistically significant difference $(P<$ $.001)$ in the enhancement values between the normal pituitary gland and the microadenoma could be consistently noted throughout all the time points measured from 60 seconds (T1) to 140 seconds (T8) (Fig 3).

\section{Microadenomas}

Of the 27 microadenomas, 7 were $6-9$ $\mathrm{mm}, 19$ were $3-5 \mathrm{~mm}$, and 1 was $<3 \mathrm{~mm}$.

\section{DISCUSSION}

In this study, we have successfully demonstrated the role of a radial $3 \mathrm{D}$ gradient-echo acquisition with GRASP reconstruction to provide a quantitative assessment of permeability characteristics and enhancement patterns of the normal pituitary gland and microadenomas.

Golden-angle radial sparse parallel MR imaging is a volumetric dynamic imaging technique based on a 3D gradient-echo sequence with radial "stack-of-stars" $k$-space sampling and goldenangle ordering. Such an image acquisition allows excellent spatial and temporal resolution. The current study exploited the potential of this technique in evaluating the pituitary gland with an in-plane resolution of $0.7 \mathrm{~mm}$, a contiguous section thickness of $0.8 \mathrm{~mm}$, and a temporal resolution of 20 seconds.

Our ROI-based analysis of STCs demonstrates a greater maximum and mean enhancement in the anterior pituitary gland compared with the posterior pituitary gland and median eminence. Furthermore, we found faster wash-in in the median eminence and posterior pituitary gland compared with the anterior pituitary gland (Fig 2). These findings may reflect differences in the underlying vascular anatomy and perfusion characteristics of these regions. The superior hypophyseal artery is a branch of the supraclinoid segment of the internal carotid artery. The inferior hypophyseal artery is a branch of the meningohypophyseal trunk that arises from the cavernous segment of the internal carotid artery. Both the superior and inferior hypophyseal arteries anastomose with each other and their counterparts at the level of the median eminence to form the primary plexus. This primary plexus of capillaries gives rise to a series of short and long portal hypophyseal veins, which pass down the stalk to form a secondary plexus to supply the anterior pituitary gland. In contrast, the posterior pituitary gland is supplied directly by the inferior hypophyseal arteries. It is likely that the differences in the blood supply to the anterior and posterior pituitary gland reflect the variation in their enhancement patterns. Specifically, the posterior pituitary gland, due to the direct vascular supply from the inferior hypophyseal artery, enhances earlier than the anterior pituitary gland. ${ }^{11}$ Similarly, the anterior pituitary gland receives blood supply from both the superior and inferior hypophyseal arteries via the portal plexus. The anterior pituitary gland, therefore, exhibits delayed wash-in, likely due to the additional time taken for the blood to course through the portal plexus of veins. Likewise, the greater maximum enhancement within the anterior pituitary gland results from an intrinsic attenuated and complex capillary network contributed by the dual vascular supply of the superior and inferior hypophyseal arteries. ${ }^{12,13}$

Our findings are supported by a previous study by Tien, ${ }^{13}$ which obtained STCs from 1.5T sagittal dynamic contrast-enhanced T1-weighted gradient-echo sequences, similarly demonstrating slightly delayed enhancement of the anterior pituitary 


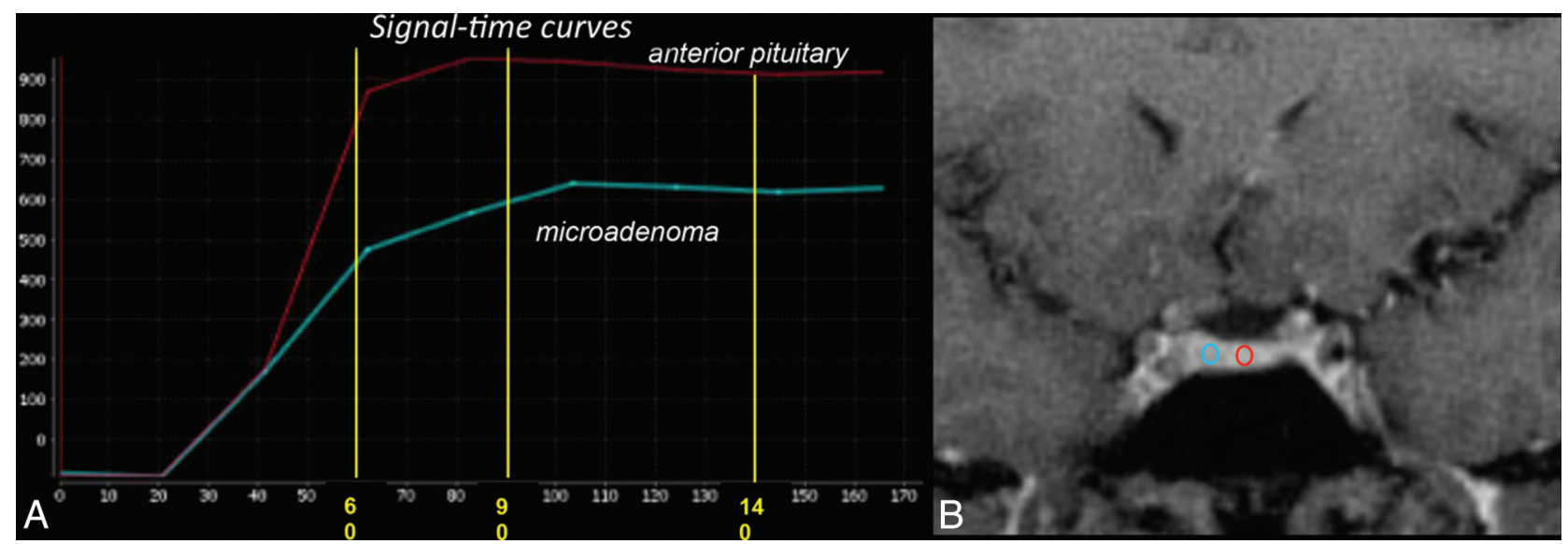

FIG 3. $A$, GRASP image demonstrates placement of ROIs within the normal-appearing pituitary gland and microadenoma. $B$, Corresponding signal-time curves are shown. Peak enhancement from the anterior pituitary gland is seen at approximately 80 seconds with subsequent gradual washout. Peak enhancement from the microadenoma is seen at approximately 100 seconds with a subsequent plateau. There is a significant difference in the enhancement between the anterior pituitary gland and the microadenoma from 60 seconds onward.

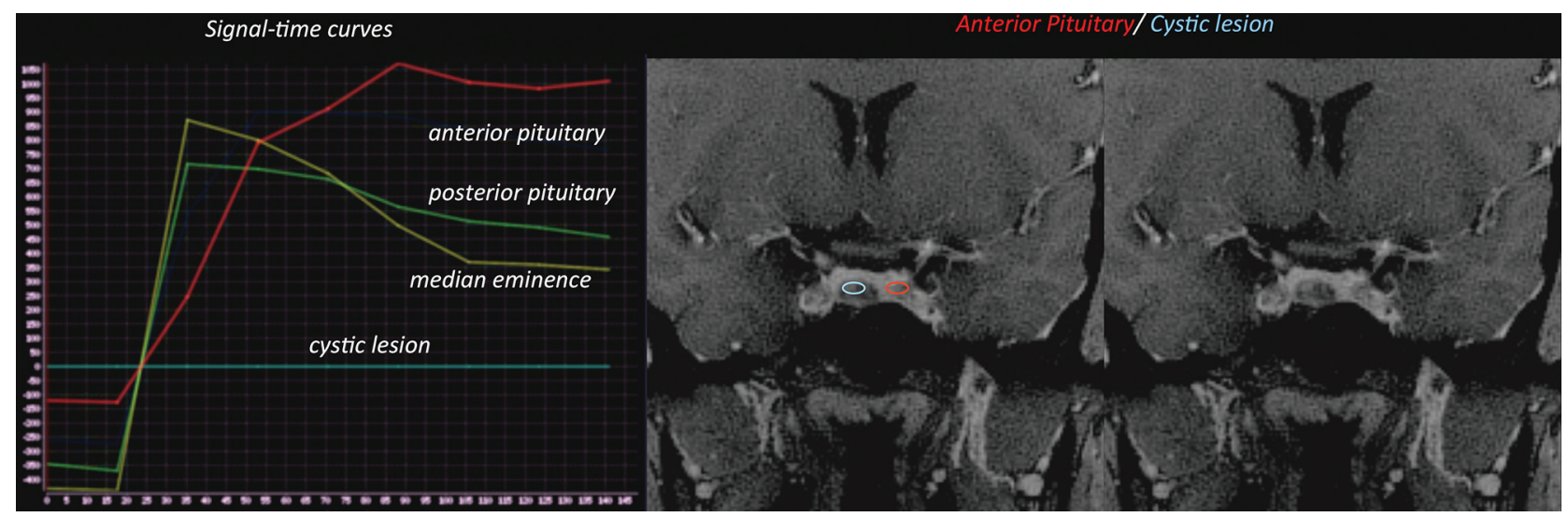

FIG 4. Signal-time curve derived from an ROI applied to a cyst demonstrates a flat STC compared with the normal STCs from the anterior pituitary gland, posterior pituitary gland, and median eminence evaluated in the same patient.

gland compared with the posterior pituitary gland and median eminence.

There is a large variation in the acquisition time (150-240 seconds) for dynamic imaging of the pituitary gland among imaging centers. ${ }^{4-6}$ Our study based on the STCs sought to establish a more defined acquisition time for dynamic pituitary gland imaging. Our results demonstrate that maximum enhancement of the anterior pituitary gland occurs at approximately $80 \pm 10 \mathrm{sec}-$ onds, subsequent to which there is no significant change in enhancement for a measured time period up to 140 seconds. In contrast, the microadenomas demonstrate maximum enhancement at $90 \pm 10$ seconds, subsequent to which there is no significant change in the enhancement for a total measured time of 140 seconds. Furthermore, statistical analysis demonstrated that a significant difference in the enhancement curves between the anterior pituitary gland and the microadenoma is seen beginning 60 seconds postinjection of contrast and continuing for the entire remaining duration of the study. Our results therefore suggest that 120 seconds ( 2 minutes) is a reasonably appropriate dynamic acquisition time to evaluate the pituitary gland following contrast administration. According to our literature search, ours is the first imaging study that has looked into the signal-time curves to establish a defined acquisition imaging time for pituitary studies.
This has significant bearing in the current economic imaging scenario, allowing appropriate use of magnet scanning time to achieve optimum patient throughput without compromising patient care. By establishing a 120-second acquisition time, we save $30-120$ seconds of imaging time from that quoted in the literature. $^{4-6}$

The study also demonstrated that the signal-time curves for microadenomas are distinctly different from those of cysts. The STC from a microadenoma demonstrated some uptake of contrast in the initial 60 seconds following contrast administration before plateauing off. In contrast, the simple cyst did not pick up any contrast and is represented by a flat STC (Fig 4).

The retrospective nature of the study, the small sample size, and lack of surgical confirmation for microadenomas are the major limitations of our study. We excluded cystic and hemorrhagic adenomas from our study population; this exclusion can be considered a relative limitation as well. However, we did this to have a homogeneous patient-microadenoma population, keeping in mind that this was a proof-of-concept study.

\section{CONCLUSIONS}

We have demonstrated that dynamic imaging of the pituitary gland by using a radial VIBE with GRASP is feasible in clinical 
practice and enables both quantitative and qualitative assessment of temporal variance in signal-enhancement patterns of the anterior and posterior pituitary gland and the median eminence. Moreover, we have shown that an acquisition time of 120 seconds following contrast administration is sufficient to provide adequate dynamic evaluation of the pituitary gland, allowing optimal use of magnet scanning time.

Disclosures: Kai Tobias Block—UNRELATED: Patents (planned, pending or issued): Patent application for GRASP technique submitted by New York University. * Fernando Boada—RELATED: Grant: National Institutes of Health. * * Money paid to the institution.

\section{REFERENCES}

1. Ezzat S, Asa SL, Couldwell WT, et al. The prevalence of pituitary adenomas: a systematic review. Cancer 2004;101:613-19

2. Rand T, Lippitz P, Kink E, et al. Evaluation of pituitary microadenomas with dynamic MR imaging. Eur J Radiol 2002;41:131-35

3. Kucharczyk W, Bishop JE, Plewes DB, et al. Detection of pituitary microadenomas: comparison of dynamic keyhole fast spin-echo, unenhanced, and conventional contrast-enhanced MR imaging. AJR Am J Roentgenol 1994;163:671-79

4. Gao R, Isoda $\mathrm{H}$, Tanaka $\mathrm{T}$, et al. Dynamic gadolinium-enhanced MR imaging of pituitary adenomas: usefulness of sequential sagittal and coronal plane images. Eur J Radiol 2001;39:139-46

5. Bartynski WS, Lin L. Dynamic and conventional spin-echo MR of pituitary microlesions. AJNR Am J Neuroradiol 1997;18:965-72
6. Lee HB, Kim ST, Kim HJ, et al. Usefulness of the dynamic gadolinium-enhanced magnetic resonance imaging with simultaneous acquisition of coronal and sagittal planes for detection of pituitary microadenomas. Eur Radiol 2012;22:514-18

7. Chadarana $H$, Block $T K$, Rosenkrantz $A B$, et al. Free-breathing radial 3D fat-suppressed T1-weighted gradient-echo sequence: a viable alternative for contrast-enhanced liver imaging in patients unable to suspend respiration. Invest Radiol 2011;46:648-53

8. Winkelmann S, Schaeffter T, Koehler T, et al. An optimal radial profile order based on the golden ratio for time-resolved MRI. IEEE Trans Med Imaging 2007;26:68-76

9. Chandarana H, Feng L, Block TK, et al. Free-breathing contrastenhanced multiphase MRI of the liver using a combination of compressed sensing, parallel imaging, and golden-angle radial sampling. Invest Radiol 2013;48:10-16

10. Feng L, Grimm R, Tobias Block K, et al. Golden-angle radial sparse parallel MRI: combination of compressed sensing, parallel imaging, and golden-angle radial sampling for fast and flexible dynamic volumetric MRI. Magn Reson Med 2014;72:707-17

11. Castillo M. Pituitary gland: development, normal appearances, and magnetic resonance imaging protocols. Top Magn Reson Imaging 2005; 16:259-68

12. Aron DC, Findling JW, Tyrrell JB. Hypothalamus and pituitary. In: Greenspan FS, Strewler GJ, eds. Basic \& Clinical Endocrinology. 5th ed. Stamford, Connecticut: Appleton \& Lange; 1997:95-156.

13. Tien RD. Sequence of enhancement of various portions of the pituitary gland on gadolinium-enhanced MR images: correlation with regional blood supply. AJR Am J Roentgenol 1992;158:651-54 